\title{
Evaluation of mid-trimester abortion: A way forward
}

\author{
Padhye SM', Karki $C^{2}$
}

'Saraswati M Padhye, Professor; ${ }^{2}$ Chanda Karki, Professor, Department of Obstetrics and Gynecology, Kathmandu Medical College Teaching Hospital, Kathmandu, Nepal

\begin{abstract}
:
Background: Need of second trimester abortion has been increasing because of the wide scale introduction of prenatal screening programs detecting women whose pregnancies are complicated by serious fetal abnormalities. Lack of accredited centers and skills required for mid trimester abortion have made this procedure inaccessible to rural population of Nepal.

Objectives: This study was conducted to assess correlation of uterine size measured by clinical examination and that assessed by ultrasound, foetal foot length measurement post abortion, dose of misoprostol required and induction delivery interval in medical induction and dilatation and evacuation cases.

Methods: This is a hospital based cross-sectional study conducted from April 2011 to July 2012. gestational age was assessed by clinical palpation and ultrasound in women undergoing mid trimester abortion. Standard protocol was followed for medical and surgical abortion. Fetal foot length was measured post abortion. Mean dose of misoprostol and induction abortion interval were noted.

Results: Mean age of the patients in dilatation and evacuation group and medical induction group were similar 28 $(S D=6.2)$ and $27(S D=7)$ respectively. The mean dose of misoprostol requiredwas 400 micrograms for dilatation and evacuation and 600 micrograms for medical induction. There was moderate degree of relationship between foot length measurement of the fetus and USG findings, high degree of positive relationship between size of the uterus and fetal foot length measurement.

Conclusion: Clinical estimation of period of gestation by size of the uterus correlated well with ultrasonography findings and fetal foot length measurement post abortion. The mid trimester service can thereforebe provided in the centers where sophisticated appliances like ultrasonography is not available.
\end{abstract}

Key words: Induction abortion interval, Mifepristone, Misoprostol, Mid trimester abortion.

\section{INTRODUCTION}

A ccording to the World Health Organization (WHO), abortion is defined as the expulsion or extraction from its mother of an embryo or fetus weighing 500gm or less when it is not capable of independent survival before it becomes viable ${ }^{1,2}$. Viability is now considered to be reached at 22-24 weeks of gestation. Second trimester, or mid-trimester, is a period ranging from 13 to 28 weeks of gestation, which again is subdivided into an early period between 13 and 20 weeks and a late period between 20 and 28 weeks. Of the 210 million pregnancies that occur each year more than 46 million end in induced abortions ${ }^{1,2}$. The incidence of unsafe abortion

Address for correspondence

Dr. Saraswati M. Padhye

Professor, Department Of Obstetrics and Gynaecology

Kathmandu Medical College Teaching Hospital

Sinamangal, Kathmandu, Nepal

E-mail: saraswati.padhye@gmail.com and associated mortality is mainly due to the growing population of women of reproductive age. An estimated 21.6 million unsafe abortions took place worldwide in 2008, almost all in developing countries ${ }^{1,2}$. Although the numbers of unsafe abortions have increased, the overall unsafe abortion rate remains unchanged at about 14 unsafe abortions per 1000 women aged 1544 years $^{1}$. It has been estimated that just over $40 \%$ of pregnancies worldwide are unplanned because of nonuse of contraception, contraceptive used ineffectively or method failure. Unplanned pregnancy and induction of abortion can be prevented by expanding and improving family planning services and choices, reaching out to communities and underserved population. Sexually active teenagers and unmarried women, migrants, and poor urban slum-dwellers need more attention. It is estimated that three out of four unsafe abortions could be eliminated if the need for family planning were fully met $^{1,2}$. 
Although the majority of abortions are performed in the first trimester, there is still a gradual increase in second-trimester abortion because of the wide scale introduction of prenatal screening programs detecting women whose pregnancies are complicated by serious fetal abnormalities such as cardiovascular and skeletal malformation. Worldwide mid-trimester abortion constitutes $10-15 \%$ of all induced abortions but is responsible for two-thirds of all major complications ${ }^{2}$. Kathmandu Medical College Teaching Hospital (KMCTH) was listed by the Ministry of Health $(\mathrm{MoH})$, Government of Nepal as a site for mid trimester abortion (MTA) in 2006 to cater to the services in accordance with the law of the country. Law of the country has approved the following indications for mid trimester. Upto 18 weeks of gestation in the case of rape or incest, any gestational age if a woman is in life threatening condition, she is mentally and physically ill or the fetus is abnormal and deformed. It is illegal if it is done for fetal sex selection and when abortion is performed under coercion and without the consent of the woman. In the case of minors $(<16$ yrs of age) or mental incompetence, a legal guardian must give the consent ${ }^{3,12}$.

The Government of Nepal has laid down the criteria for the site and provider selection for listed sites. There have been continuous efforts to improve the abortion service-technology in terms of effectiveness, technical ease of performance, acceptability and reduction of side effects and complications. Mid trimester surgical abortion is performed for elective abortion, miscarriagemanagement and for pregnancy termination due to fetal anomalies and maternal health conditions. However, during the last decade, medical methods for mid-trimester induced abortion have shown a considerable development and have become safe and more accessible. The use of misoprostol is now an established and highly effective method for termination of pregnancy (ToP). The aim of this study is to evaluate the mid trimester termination of abortion performed at KMCTH in terms of differences in the induction to abortion interval (IAI) in medical and surgical methods in mid trimester abortion (MTA), dose of Misoprostol required for medical induction (MI) and surgical method, and correlation between the gestational age estimated by uterine size and ultrasonography examination in comparison with the reference chart of Foot length for gestational age.

\section{METHODS}

This is a hospital based cross-sectional study conducted at KMCTH, Kathmandu, Nepal for the period of 15 months (April 2011 to July 2012). The study was approved by the Institutional Review Committee of the institution. Women undergoing induced abortion at more than twelve weeks gestation were included in the study. There were one hundred such cases that came to seek the service of second trimester abortion during the study period. These cases were either referred from other centers or directly coming on their own to KMCTH outpatient department requesting for termination of pregnancy.

Informed consent was taken from each patient. Clinical examination was done to know the period of gestation. The women were counselled and posted for MI or dilatation and evaucuation ( $D$ and $E$ ) depending upon the gestational period, clinical findings and client's choice. The client was counseled for MI if the period of gestation was more than 16 weeks and for $D$ and $E$ if it was 13 to 16 weeks size of the uterus. If she was found fit on clinical examination, blood grouping, (Rhesus) Rh typing, Hemoglobin estimation and Ultrasonography (USG) obstetric scan were done if these were not done before. Indications for MTA were within the legal framework of the Nation which are grouped under maternal causes and fetal causes. Maternal causes are:

Physical health: The health of the patient was at risk if the pregnancy is continued due to medical conditions and has been referred by the physician for the termination of pregnancy.

Mental health: Either a psychiatrist has referred the case for termination or at least three out of 11 positive responses in Nepali language were qualified to a mental health checklist in the "Client Personal Profile" (yellow form) prepared by Department of Health Services, Ministry of Health.

Rape/Incest: Termination of pregnancy was carried out if the patient requests for this on the ground of rape or Incest without asking further detail questions about rape.

Fetal causes: Intrauterine fetal death (IUFD), Fetal anomalies (FA).

Interventions or Procedures: The patient were kept at pre-operative room. General, systemic and local examinations were performed. The uterus was palpated and the level of fundus uteri was noted. Ultrasonic gestational age of the fetus was also recorded. She was informed about the method of termination going to be performed. In case of surgical D and E method, 
a capsule of Doxycycline $100 \mathrm{mg}$ and an analgesic tab Ibuprofen $400 \mathrm{mg}$ was given orally and two tablets of Misoprostol (200 mcg each) was given buccally as per the recommendation of job- guide provided by the department of Health Services, Ministry of Health ( $\mathrm{MoH})$, Nepal $^{2,12}$. Cervix was assessed after three hours for the softness, length and its readiness. If needed the doses of Misoprostol and or analgesic were repeated after three hours. Surgical procedure was carried out using the special WHO approved D and E set and using the "check-list" prepared by Department of Health Services, Government of Nepal. Doxycycline $100 \mathrm{mg}$ twice daily was continued for three days. The time taken for softening of the cervix from the start of Misoprostol and the time of uterine evacuation were recorded. Thus the induction to abortion interval time was calculated.

In the cases of MI, it was carried out as per the guideline, in which Mifepristone 200 mg was given orally. Client was admitted in the hospital to receive two tablets of Misoprostol (200 mcg each) buccally after 36 to 48 hours. Oral analgesic was routinely and adequately used. Antibiotics were not prescribed for Ml clients. If the clients had some reasons for not being admitted, Misoprostol as per the protocol was prescribed and she was given clear instruction to come to the hospital as soon as she started having pain or bleeding. Time interval of induction to abortion was calculated from the start of Misoprostol. The dose of Misoprostol required to achieve termination of pregnancy was noted in micrograms.

Confirmation of gestational period: After expulsion/ completion of $D$ and $E$, the foot- length (from the toe to the heel) of the fetus was measured in millimeter keeping the fetal foot on the transparent- measuring scale. The date of gestation was calculated from the supplied fetal foot length measurement chart (Table 1).

The finding of the foot length in relation to the gestational age calculated from her last menstrual period (LMP), from clinical examination of fundus uteri and the age of the fetus as calculated by USG were recorded and compared.

The data obtained from the study were entered and analyzed in the Microsoft Excel 2007.

\section{RESULTS}

There were 427 cases of abortion dealt with at KMCTH during the study period of which mid trimester abortion was carried out in 100 cases (23\%). Fifty one per cent of these clients were from Kathmandu valley and $49 \%$ was from outside the valley. Eighty two percent of clients came to this service site on their own while $16 \%$ of the clients were referred from other centers.

In case of medical induction mean age of the clients was $27(S D=6.2)$ and in surgical it was $28(S D=6)$ years. The parity was zero to five; mean being 1.9 and $2.3 \mathrm{in} \mathrm{Ml}$ and $D$ and $E$ respectively. Numbers of nulliparous were 22 and nine in $\mathrm{MI}$ and $\mathrm{D}$ and $\mathrm{E}$ respectively.

Fifty-seven clients underwent Medical induction (MI) and Forty-three clients underwent surgical method. Out of these 100 clients, seven clients were unmarried out of which three underwent surgical procedure.

1. Induction to abortion interval (IAI): Median inductionabortion time interval was 10 hours and 9.25 hours $(\mathrm{P}=0.23)$ in $\mathrm{Ml}$ and $\mathrm{D}$ and $\mathrm{E}$

2. Dose of Misoprostol: The mean (SD) dose of Misoprostol required was 600 micrograms $(S D=274)$ and 400 micrograms $(S D=276)$ for $\mathrm{MI}$ and $\mathrm{D}$ and $\mathrm{E}$ $(\mathrm{p}=0.0004)$ for $\mathrm{MI}$ and $\mathrm{D}$ and $\mathrm{E}$ respectively.

Table 1: Identification of gestational duration (weeks) through measurement of fetal foot length of the fetus (mm)

\begin{tabular}{|cccc|}
\hline Gestational duration (weeks) & Foot length $(\mathbf{m m})$ & Foot length (Range) & Foot length Range+ $\mathbf{1 ~ S D}(\mathbf{m m})$ \\
\hline 12 to 13 & 10 & $8-11$ & $7-13$ \\
13 to 14 & 13 & $12-14$ & $10-16$ \\
14 to 15 & 16 & $15-17$ & $13-19$ \\
16 to 17 & 23 & $21-24$ & $19-26$ \\
\hline $17-18$ & 26 & $24-27$ & $19-26$ \\
\hline
\end{tabular}

Source: Family Health Division, Department of Health Services, MoH, Government of Nepal 
3. Accuracy of gestational age assessment by uterine size and USG when compared with foot length. Out of the 100 abortions carried out, data for foot length measurement could be obtained only from 87 cases as there was no reports of USG in six cases and foot length could not be measured in seven cases due to expulsion of the fetus in the toilet in $\mathrm{Ml}$ and crushed fetal feet in cases of $D$ and E). It was found that there is strong positive relationship between estimated period of gestation by USG and age of the fetus measured by foot length measurement post abortion $(r=0.92, p<0.001)$. Likewise, there is strong positive relationship between uterine size and age of fetus $(r=0.76, p<0.001)$, so also there is strong positive relationship between uterine size and USG findings $(r=0.82, p<0.001)$. Overall, there was moderate degree of relationship between Foot length and USG findings $(r=0.5, p<0.000)$.There was high degree of positive relationship between size of the uterus versus foot length $(r=0.7, p<0.001)$. It was found moderate degree of positive relation between the size of the uterus and USG findings $(r=0.48, p$ $<0.001)$.

Table 2: Reasons for mid trimester abortion ( $N=100)$

\begin{tabular}{lc}
\hline \multicolumn{1}{l}{ Reasons for mid trimester abortion } & Number \\
Maternal & \\
Physical & 3 \\
Mental & 66 \\
Rape/Incest & 9 \\
Fetal & \\
Intrauterine fetal death & 13 \\
Fetal malformation & 9 \\
\hline
\end{tabular}

\section{DISCUSSION}

Mid-trimester abortion services were started from 2006 in KMCTH. However, this study is first of its kind in Nepal where we have analyzed finding in abortus (fetal footlength) to find out the accuracy of clinical estimation and USG estimation of gestational age of fetus. A total of 427 women were offered abortion services during that period out of which $100(23 \%)$ were in their second trimester of pregnancy. Almost equal number of women from within the Kathmandu valley (51\%) and from outside the valley (49\%) came seeking this service. The reasons for seeking abortion were maternal in $80 \%$ of the cases and only $20 \%$ fetal. The surgicalintervention was provided to $42 \%$ cases and the rest underwent medical termination.

The age of the mothers seeking abortion services ranged from less than 19 years to more than 40 years; most of the women being in the age group of 20 to 34 years (76\%). Almost more than one third of the mothers were first time pregnant (primi: $34 \%$ ) followed by second gravida (26\%) and third gravid (23\%) indicating that multiple pregnancy is less common a cause for seeking abortion. Most of the abortuses were in the gestational age of 1320 weeks. Usually women presenting with pregnancy of less than 16 weeks were offered surgical intervention whereas those above 16 weeks were medically induced. However, almost equal number of abortions among less than 18 weeks was medically (46\%) and surgically induced (43\%). Both the methods were found to be equally safe as there was no severe complication during both the methods.

As the type of intervention is determined by the gestational age, accurate estimation of gestational age is essential prior to deciding about the type of intervention. Accurate history, assessment of uterine size and ultrasonography are the usual methods of assessing the gestational age. Foot length of the abortuses were taken as a gold slandered to assess the accuracy of clinical examination and USG method for estimating period of gestation in cases undergoing MTA ${ }^{12}$. The ability of the clinical method of uterine size estimation to detect the correct gestational age was 33\%, whereas the ability of USG to do so was $40 \%$. However, the possibility of overestimating the gestational age was almost equal for uterine size estimation and USG estimation (32.5\% versus $33.3 \%$ ) whereas the tendency to underestimate was higher with uterine size compared to USG studies (36\% versus $27 \%$ ). Thus, as accurate assessment of GA is a prerequisite to decide whether to offer medical or surgical services, it is desirable that abortion seeker undergo USG before they are offered such services if possible.

The regimen Mifepristone followed by Misoprostol as recommendedwas chosen for $\mathrm{Ml}^{4}$. In our study we have used Mifepristone $200 \mathrm{mg}$ followed by Misoprostol 400 microgram buccaly after $36-48$ hours and the dose repeated three hourly maximum of five doses as per the need till the complete expulsion of the fetus and the placenta.

Mifepristone followed by Misoprostol after $36-48$ hours is found to be safe. The mean induction abortion interval (IAI) was nine to ten hours. This is the effective regimen for mid trimester medical abortion (MI) with younger women and those with a previous live birth. In one of the largest published study, mean IAI was found to be 5.9 hours for multi and 6.6 hours for nulliparous women where dose of Mifepristone was $200 \mathrm{mg}$ followed by 800 
mcg of Misoprostol vaginally which was repeated three hourly at the dose of $400 \mathrm{mcg}$ vaginally to maximum of five doses. The choice of this regimen was on the basis of WHO and RCOG protocol based on trials that included women predominantly at 20 weeks gestation or less ${ }^{5,6}$. In another study the use of Misoprostol alone has been used and found to be effective with the mean IAI of 13.069+6.21 huors $^{7}$. Shorter the induction abortion time the lesser is the morbidity ${ }^{8}$. Same regimen as ours was compared with four doses of $1 \mathrm{mg}$ gemeprostolvaginally every six hour in another study and the abortion-induction median time was found to be 8.7 and 10.8 hours $^{9}$.

In our study mean dose of Misoprostol requiredwas 600 micrograms $(S D=274)$ for the medical induction (MI) and 400 micrograms (SD=276) for D\&E. In other studies they have applied loading dose Misoprostol vaginally, sublingually, buccally and orally. Vaginal application and sublingual administration were found to have the same effect. In a study done at Kathmandu, using Misoprostol only, the induction abortion time ranged from four to thirteen hours. In another study, the second trimester abortion was carried out in Nepal at 13 to 22 weeks, based only on clinical assessment ${ }^{11}$.

It is extremely important that we strictly follow the protocol to provide the safe service and it is alsovery important to know the exact gestational period as the service providers were allowed to do $D$ and $E$ only up to 16 weeks of pregnancy after training and listing'.

\section{REFERENCES}

1. Unsafe abortion: global and regional estimates of the incidence of unsafe abortion and associated mortality in 2008, 3rd ed. Geneva, WHO, 2011.

2. Safe abortion: technical and policy guidance for health systems, $2^{\text {nd }}$ edition, Geneva, WHO, 2012.

3. Sammandari $\mathrm{G}$ et al. Implementation legal abortion in Nepal: a model for rapid scale-up of high quality care Reproductive Health 2012, 9:7 doi: 10.1186/1742-4755-9-7.

4. FIGO: Misoprostol Recommended Dosages, Weeks \& Faundes,.Int J GynecolObstet 2007; 99,SI56.

5. Allan Guttmacher Institute (1999). Sharing responsibility: Women, Society and Abortion worldwide.

6. Ashok PW, Templeton A,Wagaarachchi PT and Flett GM (2004).Midtrimester medical termination of pregnancy: a review of 1002 consecutive cases. Contraception.69,51-58

7. Saha R, Karki C: Misoprostol alone is effective for mid- trimester abortion. Journal of Kathmandu
In our study, induction abortion interval for $D$ and $E$ was found shorter than MI. This study has also shown that termination of pregnancy up to 16-18 weeks may be performed after taking good history of menstrual cycles and clinical examination for the gestational age measurement. Unavailability of the ultrasound facility need not be a barrier for safe provision of this service.

Only two trained professionals carried out the fundal height measurement, assessment of cervix, fetal foot length measurement of the abortus avoided bias associated with inter-observer variation. This was a strength of the study. Further studies are needed in a larger population to validate the finding of this study as the weakness was the small sample size ${ }^{12}$.

\section{CONCLUSION}

From this study there is a hope to counsel/ provide services even in remote/ hilly places on the basis of clinical examination even in the centers where sophisticated appliances like USG is not available, and decide whether the case is suitable for D and E or MI.

\section{ACIKNOWLEDGEMENT}

We are thankful to all the participants who consented to be enrolled in the study. We would like to acknowledge the faculties, residents, house officers, nurses and the hospital staffs supporting and facilitating this study.

Medicacal College, Vol.2, No.2, Issue 4, Apr.-Jun., 2013.pp59-62.

8. Society of Family planning research education, and leadership /contraception 84 (2011) 4-18, clinical Guidelines. Labour induction and abortion in the second trimester.

9. P.C.Ho,P.C.Blumenthal et al Misoprostol for the termination of pregnancy with a live fetus at 13 to 26 weeks. Review Article, International Journal of Gynecology and Obstetrics (2007), 99 (5178-5181).

10. Oi Sham et al, Pharmacokinetics of different routes of administration of Misoprostol, Human Reproduction Vol. 17,No.2 pp 332-336,2002

11. Sriwastava V, Bajracharya L, Thapa S Surgical abortion in second trimester: Initial experiences in Nepal. Kathmandu University Medical Journal (2010), Vol. 8, No. 2, Issue 30, (169-172).

12. Government of Nepal, MoH\&P, DoHS, Family Health Division and National Health Training Centre. Second Trimester Abortion Service, 2013,Manual for providers. 